\title{
AVALIAÇÃO DO CONSUMO ALIMENTAR E ESTADO NUTRICIONAL DE
}

\author{
GRADUANDOS EM ENFERMAGEM
}

\author{
Fabíola Pansani Maniglia ${ }^{1}$ \\ Daniele Mendonça Santos ${ }^{2}$ \\ Franciele Cristina Maria de Oliveira $^{3}$ \\ Júlio César Ribeiro ${ }^{4}$ \\ Recebido em: 12 jul. 2018 \\ Aceito em: 15 dez. 2018
}

RESUMO: A baixa qualidade da alimentação e o sedentarismo representam riscos para o acúmulo de gordura corporal e desenvolvimento de doenças já na juventude. $\mathrm{O}$ objetivo do estudo foi investigar o consumo alimentar e o estado nutricional de alunos do curso de Enfermagem por meio de atividade prática em uma disciplina curricular relacionada à nutrição. Trata-se de um estudo transversal feito com as informações obtidas durante o desenvolvimento de uma atividade da disciplina denominada "Aspectos nutricionais na assistência de enfermagem", na qual participaram 76 estudantes maiores de 18 anos. As informações nutricionais foram obtidas por meio de entrevista em pares, registro de um padrão alimentar e aplicação de um Questionário de Frequência Alimentar. Os integrantes do estudo eram na maioria mulheres $(80,3 \%)$ e a idade média da amostra foi de $25,3 \pm 7,3$ anos. Muitos indivíduos apresentaram ingestão hídrica insuficiente, a qual se correlacionou negativamente com o consumo de refrigerante $(r=-0,254 p=0,029) .48,8 \%$ da amostra estava acima do peso corporal e $75 \%$ eram sedentários. O fracionamento alimentar foi considerado satisfatório, mas o consumo de frutas e hortaliças foi abaixo das recomendações $(0,6 \pm 0,9 ; 1,7 \pm 1,2$ porções). Houve alto consumo de açúcar e gordura, identificado por itens, como o refrigerante e alimentos fritos. Acredita-se que a disciplina relacionada à nutrição, presente na grade curricular do curso, possa servir como uma importante ferramenta de estímulo a melhores hábitos de vida, partindo de práticas alimentares mais adequadas.

Palavras-chave: Consumo alimentar. Estado nutricional. Estudantes.

\section{EVALUATION OF FOOD CONSUMPTION AND NUTRITIONAL STATUS OF UNDERGRADUATE NURSING STUDENTS}

ABSTRACT: The poor quality of diet and the sedentary lifestyle increase the risk of body fat accumulation and the development of diseases as early as the youth. The objective of the study was to investigate the food consumption and the nutritional status of students of the Nursing course through practical activity in a curricular discipline related to nutrition. This is a cross-sectional study carried out with the information obtained during the development of an activity of the discipline called "Nutritional Aspects in Nursing Care", in which 76 students older than 18 years participated. The nutritional information was obtained by a paired interview, recording a food pattern and

\footnotetext{
1 Universidade de Franca.

2 Universidade de Franca.

3 Universidade de Franca.

4 Universidade de Franca.
} 
applying a Food Frequency Questionnaire. The study participants were mostly female $(80.3 \%)$ and the mean age of the sample was $25.3 \pm 7.3$ years. Many subjects presented insufficient water intake, which correlated negatively with the soda consumption $(r=-0.254 p=0.029) .48 .8 \%$ of the sample was overweight and $75 \%$ were sedentary. The number of meals was considered satisfactory, but consumption of fruits and vegetables was below the recommendations $(0.6 \pm 0.9,1.7 \pm 1.2$ portions $)$. There was high consumption of sugar and fat, identified by items such as soda and fried foods. It is believed that the discipline related to nutrition, present in the curriculum of the course, can serve as an important tool to stimulate better life habits, starting from more appropriate eating practices.

Keywords: Food consumption. Nutritional status. Students.

\section{INTRODUÇÃO}

Nos últimos anos o Brasil tem passado por uma rápida transição demográfica, epidemiológica e nutricional. Doenças agudas e infecciosas têm dado lugar às doenças crônicas provocadas especialmente pelos maus hábitos de vida (BRASIL, 2014; SCHMIDT; DUNCAN; AZEVEDO E SILVA et. al., 2011).

A baixa qualidade da alimentação, aliada ao sedentarismo, compõem atualmente as principais causas de obesidade e, consequentemente, de doenças crônicas como o diabetes mellitus e as doenças cardiovasculares (SCHMIDT; DUNCAN; AZEVEDO E SILVA et. al., 2011).

Dados apresentados pelo Vigitel no ano de 2016 mostraram que mais da metade da população brasileira se encontra com excesso de peso. A pesquisa ainda chama a atenção para o crescimento deste índice nos últimos dez anos, que passou de 42,6\% em 2006 para 53,8\% em 2016 (BRASIL, 2016).

Estudiosos afirmam que as modificações no consumo alimentar que contribuem para o ganho de peso estão relacionadas ao aumento do consumo de alimentos fora de casa, como em restaurantes, padarias, redes de fast food, dentre outros locais. Além disso, muitas vezes este padrão de consumo alimentar está associado à ingestão de produtos predominantemente ultra processados, os quais estão intimamente ligados ao desenvolvimento das doenças crônicas (BEZERRA; MOREIRA; CAVALCANTE, 2017; LOUZADA; MARTINS; CANELLA, 2015).

Pesquisadores afirmam que esta característica de consumo é motivada pela economia de tempo na organização do armazenamento e preparo dos alimentos e afirmam que esta realidade é bastante evidente em indivíduos em fase universitária, quando as atribulações da vida estudantil, associadas a mudanças sociais, dificultam a possibilidade de uma alimentação mais natural e saudável (LOUZADA; MARTINS; CANELLA, 2015, DALIENS; CLARYS; DE BOURDEAUDHUJI, 2014).

É importante considerar que universitários da área da saúde devam conhecer a importância de uma alimentação de boa qualidade na prevenção de doenças e manutenção da saúde, especialmente, porque vez ou outra, enfrentarão questões nutricionais de seus 
pacientes. Devido a esta realidade e até mesmo à necessidade de repensar o próprio comportamento alimentar, cursos de enfermagem têm introduzido alguma disciplina da área da nutrição na formação de seus alunos (FERESIN; SONZOGNO, 2007).

Com base nos fatos abordados acima, o presente estudo tem como objetivo investigar o consumo alimentar e o estado nutricional de alunos do curso de Enfermagem por meio de atividade prática em uma disciplina curricular relacionada à nutrição.

\section{MATERIAL E MÉTODOS}

Este trabalho foi realizado com estudantes do curso de Enfermagem da Universidade de Franca e foi aprovado pelo Comitê de Ética em Pesquisa desta mesma instituição de acordo com o Processo número 83061718.9.0000.5495 em março de 2018.

A pesquisa contou com uma amostra de conveniência formada por 76 voluntários, sendo 61 mulheres e 15 homens, com idade entre 18 e 45 anos. Todos os sujeitos assinaram Termo de Consentimento Livre e Esclarecido, autorizando a disponibilização dos seus dados para a realização do estudo.

Trata-se de um estudo transversal feito com as informações obtidas durante o desenvolvimento de uma atividade da disciplina denominada "Aspectos nutricionais na assistência de enfermagem". Os alunos foram alocados em duplas e orientados a realizarem entrevistas com seus pares de forma a obterem informações nutricionais por meio do registro de um padrão alimentar e da aplicação de um Questionário de Frequência Alimentar. Os registros alimentares foram então calculados com o auxílio do software DietPro versão 5i para que se pudesse estimar o consumo energético e de macronutrientes e, posteriormente, compará-lo às necessidades e recomendações diárias de ingestão das Dietary Reference Intakes (DRI) (INSTITUTE OF MEDICINE, 2002).

Quanto aos dados antropométricos, foram anotados os valores de peso e estatura dos integrantes do estudo para a obtenção do Índice de Massa Corporal (IMC), o qual foi classificado de acordo com as faixas propostas pela World Health Organization (WORLD HEALTH ORGANIZATION, 1997). Também foram questionados os hábitos urinário e intestinal, bem como a ingestão hídrica e a prática de exercícios físicos. Vale mencionar que para uma possível identificação de constipação intestinal, adotou-se os Critérios de Roma III, disponibilizados pela Organização Mundial de Gastroenterologia (WORLD GASTROENTEROLOGY ORGANISATION, 2010).

Posteriormente, todos os dados foram tabulados para a realização da análise descritiva por meio da obtenção dos valores de média, desvio-padrão, mediana, mínimo e máximo e análise estatística. Para a verificação de associação entre as variáveis foi empregado o Teste de correlação de Pearson e para avaliar a diferença entre grupo, aplicou-se o Teste ANOVA (Análise de variância) com post hoc de Bonferroni. O nível de significância considerado foi 0,05 . 


\section{RESULTADOS}

Durante a obtenção das informações utilizadas no presente estudo foi possível notar a dificuldade dos alunos em quantificar o próprio consumo alimentar, bem como a ingestão hídrica. A respeito da ingestão de água, especificamente, foi encontrada uma correlação positiva entre a mesma e a realização de atividade física $(r=0,355 \quad p=0,002)$ e uma correlação negativa com consumo de refrigerante $(r=-0,254 p=0,029)$.

A ingestão de água não se correlacionou com o trânsito intestinal, que assim como o hábito urinário, foi difícil de ser caracterizado pela falta de costume dos alunos em os observarem. Boa parte dos integrantes do estudo referiram hábito intestinal normal, mas não sabiam caracterizar a frequência e o aspecto das fezes. Dentre aqueles indivíduos que sabiam descrever o trânsito intestinal, verificou-se que 17 apresentavam constipação intestinal, caracterizada por menos de três movimentos intestinais por semana, esforço evacuatório e presença de fezes fragmentadas ou endurecidas.

Sobre os dados antropométricos de peso e estatura, estes foram facilmente apontados pelos indivíduos.

A Tabela 1 resume os principais dados de caracterização dos participantes do estudo.

Tabela 1. Caracterização da população do estudo $(n=76)$. Franca - SP, 2017.

\begin{tabular}{lll}
\hline Variável & $\mathbf{N}$ & $\%$ \\
\hline Sexo & & \\
Feminino & 61 & 80,3 \\
Masculino & 15 & 19,7 \\
& & \\
Idade & 50 & 65,8 \\
18 a 25 anos & 15 & 19,7 \\
26 a 34 anos & 11 & 14,5 \\
33 a 45 anos & & \\
& & \\
Exercício físico & 19 & 25,0 \\
Sim & 57 & 75,0 \\
Não & & \\
Classificação do estado nutricional pelo IMC & 9 & 11,8 \\
Baixo peso & 30 & 39,4 \\
Eutrofia & 25 & 32,9 \\
Sobrepeso & 12 & 15,9 \\
Obesidade & & \\
Porcentagem de adequação da ingestão hídrica & 22 & 28,9 \\
0 a 50\% & 33 & 43,4 \\
51 a $90 \%$ & 18 & 23,7 \\
91 a $120 \%$ & 3 & 4,0 \\
$>120 \%$ & & \\
\hline
\end{tabular}

A média e o desvio padrão da idade dos alunos foram $25,3 \pm 7,3$ anos.

Dentre os 19 indivíduos que praticavam algum exercício físico, a frequência variou entre 1 e 7 vezes na semana, sendo o valor da mediana correspondente a 3. 
Sobre os dados dietéticos, a Tabela 2 apresenta os valores estimados de energia e macronutrientes, bem como das porções de fruta e hortaliça consumidas pelos participantes. Vale ressaltar que de acordo com os valores estimados de gasto energético e consumo calórico, apenas 5 indivíduos apresentaram uma porcentagem de adequação destes valores entre 95 e 105\%.

Com relação ao número de refeições consumidas, obteve-se um valor médio diário de $5,2 \pm 1,7$, sendo que $11,8 \%$ dos indivíduos faziam 2 ou 3 refeições diárias, enquanto $48,7 \%$ apresentavam um fracionamento de 4 ou 5 refeições e 39,5\% faziam 6 ou mais refeições diárias.

O consumo diário de refrigerante foi relatado por $27,6 \%$ dos integrantes do estudo e $31,6 \%$ afirmaram consumir alimento frito diariamente.

Tabela 2. Valores estimados do consumo alimentar diário dos participantes da pesquisa $(n=76)$. Franca SP, 2017.

\begin{tabular}{llllll}
\hline Variável & Média & DP & Mínimo & Mediana & Máximo \\
\hline Energia (kcal) & 1899,0 & 840,2 & 907,0 & 1684,2 & 3408,0 \\
Energia/kg/dia (kcal) & 28,1 & 13,6 & 11,3 & 25,0 & 59,8 \\
Carboidratos (\%) & 50,5 & 11,0 & 39,4 & 51,2 & 71,8 \\
Lipídios (\%) & 31,0 & 8,7 & 19,6 & 29,9 & 37,0 \\
Proteínas (\%) & 18,5 & 6,3 & 8,6 & 17,0 & 24,8 \\
Proteína/kg/dia (g) & 1,2 & 0,7 & 0,4 & 1,1 & 3,5 \\
Fruta (porção) & 0,6 & 0,9 & 0,0 & 0,0 & 5,0 \\
Hortaliça (porção) & 1,7 & 1,2 & 0,0 & 2,0 & 5,0 \\
\hline
\end{tabular}

Ao separar os indivíduos pelo estado nutricional em 4 grupos (subnutrido, eutrófico, sobrepeso e obeso) observou-se diferença estatisticamente significativa na variável energia/kg/dia entre os grupos eutrófico e sobrepeso $(p=0,047)$ e eutrófico e subnutrido $(p=0,001)$. Também houve diferença entre todos os grupos quando avaliada a razão gasto energético/kg peso.

\section{DISCUSSÃO}

Os estudantes de Enfermagem que integraram a pesquisa eram majoritariamente jovens adultos do sexo feminino, que se encontravam com excesso de peso. Gasparetto e Silva (2012) realizaram um estudo com 112 universitários da área da saúde e verificaram que aqueles pertencentes ao curso de Enfermagem, também predominantemente do sexo feminino e com idade semelhante à da presente amostra, apresentavam maiores valores do Índice de Massa Corporal (IMC), comparado aos estudantes dos cursos de Nutrição, Fisioterapia e Educação Física. Os autores ainda destacaram que o aumento do IMC 
nesses indivíduos tende a se estender na vida profissional, merecendo atenção e intervenção adequadas (GASPARETTO; DA COSTA E SILVA, 2012).

Sabe-se que o aumento do peso corporal pode estar associado ao nível de atividade física. No presente estudo, apenas $25 \%$ dos participantes eram fisicamente ativos e a maioria praticava exercícios 3 vezes na semana. Além disso, percebeu-se que quando separados pelo estado nutricional, todos os grupos (subnutrido, eutrófico, sobrepeso e obeso) diferiram quanto à razão gasto energético por peso corporal. Um estudo realizado por graduandos em Enfermagem, publicado em 2014, investigou a prática de exercício físico por estudantes universitários de diferentes cursos e encontrou que $77,2 \%$ dos sujeitos eram sedentários (DOS SANTOS; BRITO; NETO et al., 2014). O alto índice de sedentarismo também foi evidenciado por Marcondelli e colaboradores (2008) ao avaliarem os hábitos de vida de universitários da área da saúde. Os autores constataram ainda que os cursos de Enfermagem e Medicina tinham os maiores valores de IMC e as maiores taxas de inatividade física, e que as principais justificativas para a baixa prática de exercícios foram a falta de tempo, seguida dos recursos financeiros insuficientes (MARCONDELLI; COSTA; SCHMITZ; 2008). Além do gasto energético, promovido pelo exercício, a alimentação também deve ser considerada como fator primordial e determinante do estado nutricional.

A dificuldade em quantificar a alimentação pelos estudantes avaliados no presente estudo é uma situação bastante discutida pelos pesquisadores da área de investigação do consumo alimentar. No trabalho de revisão de Fisberg, Marchioni e Colucci (2009) são apresentados diferentes motivos pelos quais o entrevistado não consegue descrever com precisão as informações sobre sua alimentação. $O$ esquecimento e a omissão proposital dos alimentos considerados menos saudáveis são muitas vezes apontados como os principais erros na identificação do consumo alimentar (FISBERG; MARCHIONI; COLUCCI, 2009).

O número médio de refeições realizadas pelos estudantes no presente estudo pareceu satisfatório, especialmente quando comparado aos achados de Loureiro (2016) ao realizar uma revisão bibliográfica sobre o estado nutricional e os hábitos alimentares de universitários. Os dados levantados pela pesquisadora mostraram que apenas $5 \%$ de um grupo de estudantes declararam fazer seis refeições por dia, sendo que a maioria relatou fazer quatro refeições. Em outro estudo analisado por Loureiro (2016), cerca de 60\% dos acadêmicos informaram não realizar as três refeições principais (café da manhã, almoço e jantar) diariamente (LOUREIRO, 2016).

A respeito da análise quantitativa da alimentação dos indivíduos, esta mostrou que apesar do valor médio do consumo energético ser condizente com as características desta população, quando analisada a adequação entre o gasto e o consumo energético, apenas 5 participantes apresentaram porcentagens entre 95 e 105\%. Também foi possível notar que o consumo energético dos indivíduos eutróficos foi significativamente diferente daqueles classificados como subnutrido ou sobrepeso, como era de se esperar.

Estudiosos apontam que apesar do entendimento básico sobre nutrição, por serem 
estudantes universitários da área da saúde, muitas vezes o valor energético consumido não está de acordo com as necessidades reais do indivíduo, o que pode comprometer o seu estado nutricional, levando à perda ou ao ganho ponderal (SOAR; DE SOUZA E SILVA; LIRA, 2012).

Já os macronutrientes também tiveram valores médios adequados quanto à porcentagem de distribuição, segundo as Dietary Reference Intakes (DRI) (INSTITUTE OF MEDICINE, 2002). Mas quando analisada a qualidade da alimentação, por meio do consumo de frutas e hortaliças, percebeu-se que a ingestão destes itens foi abaixo da recomendação do Guia Alimentar para a População Brasileira (BRASIL, 2014).

Carneiro e colaboradores (2016) investigaram o consumo alimentar habitual de alunos da Universidade Federal do Pará e encontraram valores correspondentes a

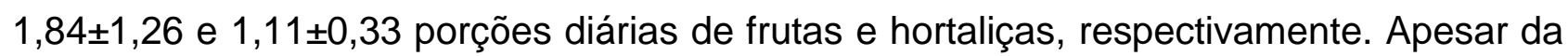
ingestão destes vegetais ter sido superior à verificada no presente estudo, esta ainda se encontra insuficiente (CARNEIRO; LIMA; MARINHO, 2016).

Já o consumo diário de refrigerante foi referido por uma parcela importante da amostra avaliada, assim como a ingestão de alimentos fritos todos os dias. Práticas alimentares como estas são amplamente verificadas no ambiente universitário e fazem com que se exceda a quantidade máxima permitida para o consumo de açúcares simples e gorduras de adição (BRASIL, 2014; LOUREIRO, 2016). Ainda sobre o refrigerante, foi verificada uma correlação inversa do seu consumo com a ingestão de água pelos estudantes, dos quais $72,3 \%$ atingiram no máximo $90 \%$ de adequação da ingestão hídrica. Por meio de uma revisão sistemática, pesquisadores constataram esta mesma relação entre a alta ingestão de bebidas açucaradas e a baixa ingestão de água e os mesmos ainda enfatizaram a necessidade de promover palestras e atividades educativas sobre o assunto para o publico infantil, já que nos adolescentes e adultos parece ser mais difícil atingir modificações neste padrão de consumo (VARGAS-GARCIA; EVANS; PRESTWICH et al., 2017). Também pôde-se notar que os indivíduos praticantes de exercício físico ingeriam volumes diários maiores de água e que esta não esteve associada ao hábito intestinal da população. Acredita-se que a maior ingestão hídrica nos alunos fisicamente ativos seja um fato justificado pelo aumento da transpiração, provocado pelo exercício, que leva a uma necessidade de reposição da água corporal perdida (MORA-RODRIGUEZ; ORTEGAF. FERNANDEZ-ELIAS, 2016).

É importante destacar que os estudiosos confirmam a substituição de alimentos naturais, como os vegetais, por itens industrializados não só entre estudantes universitários, mas também na população brasileira em geral, e apontam as doenças crônicas, especialmente decorrentes da obesidade, como o principal risco em adotar este hábito (LOUZADA; MARTINS; CANELLA, 2015; LOUREIRO, 2016). 


\section{CONSIDERAÇÕES FINAIS}

Os dados do presente estudo permitiram concluir que os alunos de Enfermagem avaliados eram predominantemente sedentários, encontravam-se acima do peso corporal e apresentavam baixo consumo de frutas e hortaliças, ingestão hídrica insuficiente e consumo elevado de açúcares e gordura.

Acredita-se que a disciplina relacionada à nutrição, presente na grade curricular do curso, possa servir como uma importante ferramenta de estímulo a melhores hábitos de vida, partindo de práticas alimentares mais adequadas.

\section{REFERÊNCIAS}

BEZERRA, llana Nogueira; MOREIRA, Tyciane Maria Vieira; CAVALCANTE, Jessica Brito. et. al. Alimentação fora do lar no Brasil segundo locais de aquisição. Rev Saúde Pública, v. 51, n. 15, p. 1-8, 2017.

BRASIL. Ministério da Saúde. Secretaria de Atenção à Saúde. Departamento de Atenção Básica. Guia alimentar para a população brasileira / Ministério da Saúde, Secretaria de Atenção à Saúde, Departamento de Atenção Básica. - 2. ed. - Brasília: Ministério da Saúde, 2014.

BRASIL. Ministério da Saúde. Secretaria de Vigilância em Saúde. Departamento de Vigilância de Doenças e Agravos Não transmissíveis e Promoção da Saúde. VIGITEL Brasil 2016: vigilância de fatores de risco e proteção para doenças crônicas por inquérito telefônico. Brasília: Ministério da Saúde; 2016.

CARNEIRO, Maria de Nazareth de Lima; LIMA, Priscyla Souza de; MARINHO, Lorrany Miranda. et al. Estado nutricional de estudantes universitários associados aos hábitos alimentares. Rev Soc Bras Clin Med, v. 14, n. 2, p. 84-8, 2016.

DELIENS, Tom; CLARYS, Peter; DE BOURDEAUDHUJI, Ilse. et al. Determinants of eating behaviour in university students: a qualitative study using focus group discussions. BMC Public Health, v. 14, n. 53, p. 1-12, 2014.

DOS SANTOS, Leianny Rodrigues; BRITO, Emmanuel Calisto da Costa; NETO, José Cláudio Garcia Lira. et al. Análise do sedentarismo em estudantes universitários. Rev enferm UERJ, v. 22, n. 3, p. 416-21, 2014.

FERESIN, Cátia; SONZOGNO, Maria Cecília. Reflexões sobre a inserção da disciplina de nutrição na formação do enfermeiro. Rev Latino-am Enfermagem, v. 15, n. 6, 2007.

FISBERG, Regina Mara; MARCHIONI, Dirce Maria Lobo; COLUCCI, Ana Carolina Almada. Avaliação do consumo alimentar e da ingestão de nutrientes na prática clínica.

Arq Bras Endocrinol Metab, v. 53, n. 5, p. 617-624, 2009.

GASPARETTO, Rosa Maria; DA COSTA E SILVA, Rosana Carolo. Perfil antropométrico dos universitários dos cursos de nutrição, enfermagem, fisioterapia e educação física do Centro Universitário La Salle, Canoas/RS. Rev. Assoc. Bras. Nutr, v. 4, n. 5, p. 29-33, 
2012.

INSTITUTE OF MEDICINE. Food and Nutrition Board. Energy. In: Institute of Medicine. Food and Nutrition Board, ed. Dietary Reference Intakes - Energy, carbohydrate, fiber, fat, fatty acids, cholesterol, protein, and amino acids. Washington (DC): The National Academy Press; 2002. p.107-264.

LOUREIRO, Marina Paraluppi. Estado nutricional e hábitos alimentares de universitários. Segurança Alimentar e Nutricional, v. 23, n. 2, p. 955-972, 2016.

LOUZADA, Maria Laura da Costa; MARTINS, Ana Paula Bortoletto; CANELLA, Daniela Silva. et al. Alimentos ultraprocessados e perfil nutricional da dieta no Brasil. Rev Saúde Pública, v. 49, n. 38, p. 1-11, 2015.

MARCONDELLI, Priscilla; COSTA, Teresa Helena Macedo da; SCHMITZ, Bethsáida de Abreu Soares. Nível de atividade física e hábitos alimentares de universitários do $3^{\circ}$ ao $5^{\circ}$ semestres da área da saúde. Rev. Nutr., v. 21, n. 1, p. 39-47, 2008.

MORA-RODRIGUEZ, Ricardo; ORTEGA, Juan F. FERNANDEZ-ELIAS, Valentin E. et al. Influence of Physical Activity and Ambient Temperature on Hydration: The European Hydration Research Study (EHRS). Nutrients, v. 8, n. 252, p. 1-13, 2016.

SCHMIDT, Maria Inês; DUNCAN, Bruce Bartholow; AZEVEDO E SILVA, Gulnar. et al. Chronic non-communicable diseases in Brazil: burden and current challenges. Lancet, v. 377, n. 9781, p. 1949-61, 2011.

SOAR, Claudia; DE SOUZA E SILVA, Patricia; LIRA, Janaína Guarino. Consumo alimentar e atividade física de estudantes universitários da área de saúde. Revista Univap, v. 18, n. 31, p. 41-47, 2012.

VARGAS-GARCIA, E.J; EVANS, C.E.L; PRESTWICH, A. et al. Interventions to reduce consumption of sugar-sweetened beverages or increase water intake: evidencefrom a systematic review and meta-analysis. Obesity Reviews, v. 18, 1350-1363, 2017.

WORLD GASTROENTEROLOGY ORGANISATION (WGO). WGO Practice Guidelines: Constipação: uma perspectiva mundial. 2010. Disponível em: < http://www.worldgastroenterology.org/UserFiles/file/guidelines/constipation-portuguese2010.pdf>.

WORLD HEALTH ORGANIZATION (WHO). Obesity: Preventing and managing the global epidemic. Geneve; 1997. 